\title{
Aromatherapie: Mit der Kraft der Natur
}

\author{
Übelkeit und Kreislaufbeschwerden im Griff Ätherische und fette Öle sowie \\ Pflanzenwässer (Hydrolate) werden seit Jahrzehnten von Hebammen empfohlen. \\ Schwangere Frauen nutzen Aromamischungen erfolgreich in der Selbstbehand- \\ lung als unterstützende Maßnahme um Beschwerden zu lindern. Beispielhaft \\ sind hier Übelkeit und Kreislaufbeschwerden aufgeführt. Ingeborg Stadelmann
}

I mmer mehr Schwangere suchen den Rat einer Hebamme. Die Erwartungshaltung im Bereich der komplementären Methoden, zu der auch die Aromatherapie zählt, nimmt stetig zu. Viele Beschwerden in einer Schwangerschaft sind meist physiologisch und bedürfen eigentlich überhaupt keiner Therapie. Jedoch kann eine rechtzeitige präventive naturheilkundliche Begleitung Pathologie verhindern. Das eine oder andere Ziehen in der Leiste oder im Kreuzbeinbereich oder die Einschlafprobleme müssen wohl sein, damit sich die Frau immer wieder ins Bewusstsein rufen kann: Wenn dieses Kind geboren ist, wird mein Alltag ein anderer werden. Sie hat also viele Wochen Zeit, sich durch körperliche Veränderungen daran zu gewöhnen, dass sie (wieder) Mutter wird. In der Hebammenhilfe wird nicht immer nur Rat für solche Beschwerden gesucht, oftmals erfahren wir in solchen Gesprächen von verborgenen Ängsten oder Problemen.

\section{Mit Düften gegen die Übelkeit}

Früher galt das erhöhte Geruchsempfinden einer Frau als das erste sichere Anzeichen für eine Schwangerschaft. Heute unterstützt die Sensibilität der Nase mit dem gezielten Einsatz ätherischer Öle und Mischungen das Wohlbefinden während der gesamten Schwangerschaft. Die Pflanzenwelt reguliert mit ihren Duftstof-

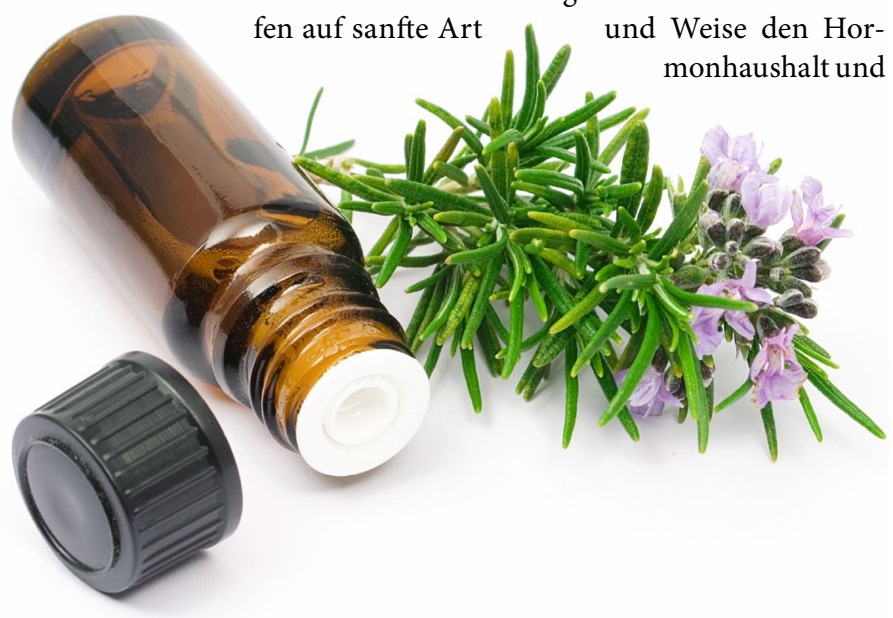

hilft so, normale Beschwerden entspannt anzunehmen und sie nicht zu pathologisieren.

Ursache von Schwangerschaftsübelkeit ist meist eine erhöhte Produktion des Schwangerschaftshormons, was jedoch hinsichtlich der Schwangerschaft ein erwünschter und positiver Zustand ist. Dies sollte werdenden Müttern meines Erachtens vorrangig als Erklärung in der Hebammensprechstunde vermittelt werden. So wird eine der ersten Ängste hoffentlich in ein positives und bejahendes Thema verwandelt. Zuden sollte der Frau vermittelt werden, dass oft die typischen Schwangerschaftsgelüste vor dieser Übelkeit schützen und sie diese Begehrlichkeiten ruhig zulassen soll.

Aber auch das Absetzen von Nikotin und Koffein kann zu Entzugserscheinungen wie Übelkeit und Erbrechen führen und muss daher entsprechend therapiert werden. Aus der Schatztruhe der komplementären Medizin haben sich zusätzlich noch die klassische Homöopathie und die Akupunktur bewährt.

Ätherische Riechfläschchen sind bei Übelkeit eine uralte bewährte Methode. Die erste Wahl darf immer bei Zitrusölen liegen: Grapefruit (Citrus x paradisi), Limette (Citrus x aurantiifolia), Zitrone (Citrus limon). Die Frau kann dann bei Bedarf an ihrem Lieblingsöl einfach riechen, eine Duftlampe oder elektrischen Zerstäuber aktivieren (s. Kasten zur Dosierung). Eine weitere Variante sind Aromasprays. Hier sprüht die Anwenderin nach Bedarf ein oder zwei Sprühstöße mit etwas Abstand vor sich in die Luft. Diese Sprays sind aber immer alkoholhaltig, da sich das ätherische Öl ansonsten nicht versprühen lässt.

\section{Vorteile einer Aromamischung}

Viele Frauen bevorzugen Aromamischungen. Sie bieten den Vorteil, dass der Duft der frischen Zitrusöle länger anhält, denn es werden, entsprechend der Parfümerie, leichtflüchtige Kopfnoten mit einer zart-blumigen Herznote und meist einer schwerflüchtigen Fußnote kombiniert. Solche Duftmischungen erfordern Fingerspitzengefühl und Fachwissen.

$\mathrm{Zu}$ den genannten Zitrusölen lässt sich zum Beispiel sehr gut das zitronig asiatische Lemongras (Cymbopogon flexuosus) und 
das kostbare Öl der Blüten vom Bitterorangenbaum Neroli (Citrus aurantium) mischen. Sein intensiver blumig-frischer Duft wird immer sparsam dosiert, nicht nur wegen des Preises, sondern wegen seines dominanten Duftverhaltens in Mischungen. Neroli zählt zu den wissenschaftlich gut erforschten Ölen. Es wird häufig bei Ängsten und Sorgen eingesetzt, die manchmal gar in Panikattacken enden können, aber auch bei Schlafstörungen. Sandelholz neukaledonisch (Santalum neocaledonicum), das einst berühmte Santalum album aus Indien, stammt aus Neukaledonien, da Indien seit einigen Jahren ein Exportverbot ausgesprochen hat, um die Ressourcen zu schonen. Es riecht ebenfalls balsamisch warm, leicht süßlich und wird als fixierende Fußnote in Mischungen gering zugesetzt. Wissenschaftliche Untersuchungen zeigen außerdem, dass sein Einzelduft außerdem eine entspannende Wirkung hat.

\section{Ingwer - auch als ätherisches Öl bei Übelkeit?}

Ingwer wird als Antiemetikum in der Schwangerschaft unter den Frauen und bei Hebammen immer beliebter. Und das mit gutem Recht. Allerdings muss bei der Verwendung jedoch stets darauf geachtet werden, ob das ätherische Öl der Ingwerwurzel (Ginger officinale) gemeint ist oder ob es sich um eine phytotherapeutische Anwendung, bzw. Verarbeitung des Rhizoms handelt, denn sowohl die Anwenungs als auch Wirkweise unterscheiden sich erheblich. Grundsätzlich kann beim ätherischen Öl hinsichtlich der antiemetischen Wirkung auf keine aktuelle Studiendatenlage zurückgegriffen werden. In Studien aus den 1970er Jahren ist das konzentrierte ätherische Öl nicht exakt benannt, sondern nur die Verwendung des Rhizoms bzw. nur Extrakte des Öls (Steflitsch et al. 2013). Zudem handelt es sich um die innere Einnahme von Ingwerkapseln. Wissenswert ist jedoch, dass das Rhizom reichlich ätherisches Öl enthält, neben den nichtflüchtigen(!) Scharfstoffen (Gingerole). Für eben diese Stoffe, die auch unter dem Begriff Shigaole bekannt sind, liegen wissenschaftliche Bestätigungen vor, dass sie bei Übelkeit und Erbrechen hilfreich sind (Stahl-Biskup 2020).

Ob das ätherische Öl antiemetisch wirkt, ist also nach wie vor unerforscht, jedoch konnten in der Praxis bisher vielfach gute Er- fahrungen damit gesammelt werden. Häufig aber in der Kombination mit anderen ätherischen Ölen, um eine nasenfreundliche Duftnote zu erreichen. Zudem liegen auch keine Wirksamkeitsbelege bei der phytotherapeutischen Anwendung von Ingwer gegen Schwangerschaftsübelkeit vor. Dennoch darf Ingwerwurzelauszug in der Schwangerschaft gerne empfohlen werden (Wenigmann 2017). Diese Aussage bezieht sich jedoch nur auf den wässrigen Auszug der Ingwerwurzel und nicht auf das ätherische Öl!

Rezeptur: 0,5-1 g grob gepulverten oder geschnittenen Ingwer mit ca. $150 \mathrm{ml}$ siedendem Wasser übergießen, bedeckt fünf Minuten stehen lassen und über ein Sieb abgießen. In Studien mit schwangeren Frauen konnte gezeigt werden, dass Ingwerwurzelstock weder Missbildungen verursacht noch andere Fötus-toxische Eigenschaften aufweist (Wenigmann 2017).

\section{Rosmarinöl - ein idealer Helfer bei Kreislaufbeschwerden}

Das ätherische Öl Rosmarin (Rosmarinus officinalis Ct. Verbenon oder Ct. Cineol) mit seinem oft unbekannten aber angenehm frischkrautigen, leicht eukalyptusartigen Duft ist ein sehr bewährtes Öl bei instabilem Kreislauf - egal, ob in der Früh- oder Spätschwangerschaft oder post partum. Viele Hebammen berichten von posi-

\section{WER DARF MISCHEN?}

Hebammen dürfen für ihre Praxisräume oder in $\mathrm{Ab}$ sprache mit der Klinikleitung für die dortigen Räume einen Raumduft anmischen. Allerdings muss jedoch auf eine korrekte Beschriftung sowie Kennzeichnung mit den entsprechenden Warnhinweisen und dem tastbaren Blindendreieck deklariert sein.

Außerdem müssen die Kolleginnen im Team darüber informiert sein, dass eine solche Aromamischung nicht zur Anwendung am Menschen genutzt werden darf.

\section{f DUFTLAMPE, VERNEBLER \& DIFFUSOR - WAS IST WAS?}

Bei einer herkömmlichen Duftlampe wird eine Kerze zur Erwärmung und somit Verflüchtigung des Öls verwendet. Die Duftentfaltung ist hier dem Gerät entsprechend unterschiedlich. Das Wassergefäß sollte jedoch immer gefüllt sein, damit das ätherische Öl nicht zu heiß werden kann.

Bei den elektrischen Geräten sind folgende Begriffe zu lesen: Aroma-Vernebler, Zerstäuber, Diffuser, Diffuseur, Diffusor ... Bei allen handelt es sich um Zerstäuber (besser: Vernebler). Sie zerstäuben Flüssigkeiten, so dass ein feiner Nebel entsteht, der sich in der Luft auflöst - die Geräte müssen also auch mit Wasser gefüllt werden.

Geräte mit Düsenzerstäubung können in doppelter Hinsicht auch als "Trocken-Vernebler" bezeichnet werden, denn sie arbeiten ohne Wasser und produzieren - eine geeignete Düse vorausgesetzt - einen trockenen Nebel, der nicht kondensiert. "Diffuser" und "Diffuseur" sind die englisch- bzw. französischsprachigen Bezeichnungen - „Diffusor" ist eine Eindeutschung dazu (FORUM 2020).

\section{Die richtige Dosierung von Aromaölen}

- Raumgröße beachten

- 3-5 Tropfen der Aromamischung (bei normal großen Räumen)

_ Alle 2-3 Stunden erfolgt eine Intervallbeduftung für ca. 20 Minuten

Beachte: Das Gerät muss regelmäßig (täglich) gereinigt werden, denn alte ätherische Öl-Reste oxidieren und sind damit gesundheitsschädlich. 
tiven Erfahrungen. In der Fachliteratur finden sich reichlich wissenschaftlich nachgewiesene Wirkungen hinsichtlich seiner kreislaufanregenden und durchblutungsfördernden Wirkung. Auch hier existiert keine Wirkstoffforschung in Schwangerschaft und Stillzeit (Anm. d. Red.: Mehr zu Studien in der Aromatherapie auf S. 45) aber über 30 Jahre Erfahrung im Umgang mit hypotonen Frauen. Bei Hypertonie jedoch ist Rosmarinöl streng kontraindiziert!

Beim Einsatz von Rosmarinöl, ob als Riechfläschchen, Naturparfüm, Wasch- oder Badezusatz oder in einer Aromamischung mit fettem Pflanzenöl für eine Einreibung, muss immer auf die richtige Auswahl des Chemotyps geachtet werden.

\section{Was bedeutet "Chemotyp“ bei ätherischen Ölen?}

Ätherische Öle sind stark wirksame Pflanzenkonzentrate, die pur hautreizend und schleimhautätzend, in entsprechenden Verdünnungen aber gut verträglich sind. Diese Vielstoffgemische können aus wenigen oder bis zu mehreren Hundert Einzelsubstanzen (Kohlenwasserstoffe) bestehen. Das ätherische Öl, gewonnen mittels Wasserdampfdestillation, kann mit Hilfe modernster Analytik in seine biochemischen Wirkstoffe aufgespalten werden. So werden die winzig kleinen Moleküle am Bildschirm sicht- und auch messbar. Grundsätzlich handelt es sich um leichtflüchtige Terpene, diese wiederum werden in Wirkstoffgruppen unterteilt: die Monoterpene, die Sesquiterpene und die seltenen Di- und Triterpene. Viele dieser flüchtigen Substanzen werden sehr individuell im Geruch wahrgenommen. Manche dieser chemischen Bausteine sind toxisch. Die Chemie der ätherischen Öle ist ein eigenes Thema und muss bei Anwendung von eigenen Kreationen oder Rezepturen natürlich unbedingt beachtet werden.

Nicht alle ätherischen Öle einer botanischen Art (z.B. Rosmarinus officinalis), sind in ihren Inhaltsstoffen immer identisch. Diese schwanken je nach Ursprungsland, Bodenbeschaffenheit, Klima, Höhenlage, Erntezeitpunkt und Destillationsvorgang. Herrscht in einem ätherischen Öl ein Inhaltsstoff dominant vor, erhält das Öl entsprechend diesem chemischen Baustein eine Zusatzbezeichnung um es differenzieren zu können: den Chemotyp. Beim Rosmarin sind dies Cineol, Verbenon oder Borneon. Es sind also bei seriösen ätherisch-Ölanbietern folgende Rosmarinöle erhältlich: Rosmarin Ct. Cineol, Rosmarin Ct. Verbenon, Rosmarin Ct. Borneon (auch als Ct. Camphora bezeichnet) (Tab. 1, e-only).

Werden ätherische Öle, wie das Rosmarinöl, als Einzelöl von der Hebamme eingesetzt, hat sie unbedingt auf die richtige Variante zu achten. Für Schwangere ist der Chemotyp Verbenon zu bevorzugen, und in Ausnahmen Ct-Cinoel. Letzteres eignet sich, wenn eine Erkältung vorliegt, denn der Wirkstoff 1,8 cineol ist in seiner antibakteriellen und antiviralen als auch schleimlösenden Wirkung bestens erforscht.

Kontraindiziert in der Hebammenarbeit ist das Rosmarinöl vom Chemotyp Borneon (Ct. Camphora). Es enthält einen zu hohen Anteil von Campherbestandteilen. Diese zählen zu den kritischen Wirkstoffen für Schwangere und Babys und dürfen auf keinen Fall eingesetzt werden. Zum einen sind Campher und Öle, die Bestandteile davon enthalten, neurotoxisch und zum anderen regen sie die glatte Muskulatur (Uterus) an. Sie sind also kontraindiziert in der Schwangerschaft; auch bei Kleinkindern bis zum ca. vierten Lebensjahr ist der Einsatz zu meiden, da Kinder mit Atemdepressio- nen reagieren können. Auch für Epileptiker und Asthmatiker gilt das Öl als ungeeignet.

\section{Wirkstoffe und Wirkweisen - es gibt viele Unbekannte}

Die Wirkstoffe sind längst nicht alle im Detail in ihrer Wirkung und Eigenschaft zu zuordnen. In einem Tropfen Öl sind hunderte Substanzen. Viele Wirkungen sind bekannt, aber noch sehr wenig bis gar nicht in ihrer Wirkweise am und im Menschen erforscht. Nach wie vor sind wir auf Berichte und Beobachtungsstudien angewiesen. Die kleinen flüchtigen Moleküle der Öle, sie haben eine Größe zwischen 126 und $225 \mathrm{~g} / \mathrm{mol}$, werden nur in Verdünnungen aufgetragen oder eingenommen und sind bislang noch nicht im menschlichen Gewebe nachweisbar. Im Jahr 1992 konnte anhand der Reinsubstanzen Linalool und Linalylacetat die Aufnahme über Einatmen und die Haut sowie deren Ausscheidung über Ausatemluft, Urin und Stuhl nachgewiesen werden (Jäger et al. 1992). Es gilt zu hoffen, dass sich Hebammen im Studium auch um die angewandte Aromatherapie kümmern, damit wir in der Hebammenarbeit auf Evidenzen zurückgreifen können.

III

\section{FAZIT}

Viele Schwangerschaftsbeschwerden lassen sich mit der Aromatherapie behandeln. Für die richtige Anwendung ist Fachwissen über Wirkweise und Dosierung erforderlich, ansonsten kann es zu unerwünschten Nebenwirkungen kommen.

Bei Übelkeit helfen Aromamischungen mit Zitrusölen und Ingwerwurzel-Auszügen.

Rosmarinöl hilft bei Hypotonie - bei Hypertonie ist es jedoch kontraindiziert.

Schlüsselwörter: Aromatherapie, Rosmarin, Schwangerschaftsübelkeit, Kreislaufbeschwerden, Zitrusöle, Ingwer, Chemotyp

\& Tabelle 1 finden Sie online auf springerpflege.de und im eMag der HebammenWissen.

\& Die Literaturliste finden Sie online auf springerpflege.de und im eMag der HebammenWissen.

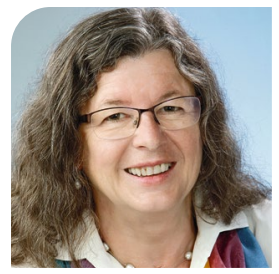

Ingeborg Stadelmann

Hebamme, Aromatherapeutin, Präsidentin FORUM Essenzia e.V., Referentin für Aromatherapie, Homöopathie, Phytotherapie und Geburtshilfe Nesso 8, 87487 Wiggensbach ingeborg.stadelmann@hebamme-stadelmann.de 